\title{
Prevalência do Hiperaldosteronismo Primário em uma Liga de Hipertensão Arterial Sistêmica
}

\author{
Prevalence of Primary Hyperaldosteronism in a Systemic Arterial Hypertension League

\begin{abstract}
Maria Jacqueline Silva Ribeiro, José Albuquerque de Figueiredo Neto, Edson Viriato Memória, Maíra de Castro Lopes, Manuel dos Santos Faria, Natalino Salgado Filho, Thiara Castro de Oliveira

Hospital Universitário Presidente Dutra da Universidade Federal do Maranhão (UFMA), São Luís, MA - Brasil
\end{abstract}

\begin{abstract}
Resumo
Fundamento: Até recentemente, o hiperaldosteronismo primário era considerado uma causa rara de hipertensão secundária. Porém, ao longo dos últimos anos, muitos estudos têm sugerido que essa doença pode afetar até $20 \%$ dos hipertensos.
\end{abstract}

Objetivo: Determinar a prevalência do hiperaldosteronismo primário em pacientes hipertensos em tratamento na liga de hipertensão de um hospital universitário.

Métodos: Foram realizadas dosagens de aldosterona sérica e atividade plasmática da renina em 105 pacientes, em vigência do tratamento anti-hipertensivo usual, excetuando-se aqueles em uso de beta-bloqueadores e espironolactona, em jejum e após repouso na posição deitada por 20 minutos. Aqueles com relação aldosterona/atividade plasmática da renina maior que 25 foram submetidos ao teste de supressão com sobrecarga salina endovenosa e, após a confirmação da autonomia da secreção de aldosterona, foi realizada tomografia computadorizada das adrenais. Os resultados são apresentados como porcentagens, médias e desvios-padrão.

Resultados: Dos 105 pacientes, 6,54\% eram hipertensos refratários. Nove apresentaram relação aldosterona/atividade plasmática da renina $>25(8,5 \%$ do total). Destes, oito foram submetidos ao teste de supressão e um (hipertenso refratário) teve o diagnóstico confirmado de hiperaldosteronismo primário $(0,96 \%$ do total). Foi realizada tomografia computadorizada de adrenais, sendo considerada normal.

Conclusão: A prevalência do hiperaldosteronismo primário na amostra estudada foi de 0,96\% do total. No entanto, quando avaliados apenas os portadores de hipertensão refratária, a prevalência foi de 14,3\%. (Arq Bras Cardiol 2009;92(1):39-45)

Palavras-chave: Hiperaldosteronismo, prevalência, hipertensão.

\begin{abstract}
Summary
Background: Until recently, primary hyperaldosteronism was considered a rare cause of secondary hypertension. However, in recent years, many studies have suggested that this disease can affect up to $20 \%$ of hypertensive individuals.

Objective: To determine the prevalence of primary hyperaldosteronism in hypertensive patients treated at the hypertension league of a university hospital.

Methods: Serum aldosterone and plasma renin activity levels were measured in 105 patients while they were undergoing standard antihypertensive treatment, with the exception of those using betablockers and spironolactone, in fasting condition and after rest in the supine position for 20 minutes. Those with an aldosterone/plasma renin activity ratio > 25 were submitted to the saline suppression test and, after the confirmation of the autonomy of aldosterone secretion, a computed tomography of the adrenals was performed. The results are presented as percentages and means and standard deviations.
\end{abstract}

Results: Of the 105 patients, 6.54\% presented refractory hypertension. Nine presented an aldosterone/plasma renin activity ratio > 25 (8.5\% of the total). Of these, 08 were submitted to the saline suppression test and 01 (with refractory hypertension) had the diagnosis of primary hyperaldosteronism confirmed (0.96\% of the total). A computed tomography of the adrenals was performed, which showed normal results.

Conclusion: The prevalence of primary hyperaldosteronism in the studied sample was $0.96 \%$ of the total. However, when only the patients with refractory hypertension were evaluated, the prevalence was 14.3\%. (Arq Bras Cardiol 2009;92(1):37-43)

Key words: Hyperaldosteronism; prevalence; hypertension.

Full texts in English - http://www.arquivosonline.com.br

Correspondência: Maria Jacqueline Silva Ribeiro •

Av. Coronel Colares Moreira, 555 - Edifício Medical Center, Sala 206 - Renascença II - 65075-441, São Luís, MA - Brasil

E-mail: jacqueribeiro.cardio@uol.com.br

Artigo recebido em 16/04/08; revisado recebido em 16/05/08; aceito em 30/05/08. 


\section{Introdução}

O hiperaldosteronismo primário (HAP) é caracterizado pela elevação dos níveis de aldosterona plasmática e urinária, supressão da atividade plasmática da renina (APR), hipertensão arterial sistêmica, hipocalemia e alcalose metabólica'.

As estimativas de prevalência do HAP que utilizaram a hipocalemia como critério diagnóstico revelaram a presença da síndrome entre $0,05 \%$ e $2 \%$ da população de hipertensos ${ }^{2}$.

No entanto, a partir de 1990, quando vários centros passaram a adotar a relação aldosterona/APR na triagem do HAP, sua prevalência aumentou para valores superiores a $12 \%{ }^{3}$.

Admite-se que esta nova estratégia corretamente aplicada à população de hipertensos poderá identificar pelo menos um entre dez indivíduos como portador de $\mathrm{HAP}^{4}$.

Diante da estimativa de que haverá um aumento na prevalência da hipertensão arterial sistêmica (HAS) em torno de $60 \%$ até 2025 , o que significa que $30 \%$ da população mundial será hipertensa ${ }^{5}$, a determinação da prevalência do HAP, uma causa de HAS passível de tratamento clínico específico orientado por mecanismos fisiopatológicos, e até curativo, torna-se fundamental para diminuirmos as complicações decorrentes dessa doença.

Esse estudo foi desenhado, então, para determinar a prevalência do HAP em pacientes hipertensos em tratamento na liga de hipertensão de um hospital universitário.

\section{Métodos}

A pesquisa foi realizada na Liga de HAS de um hospital universitário. Para o cálculo da amostra, utilizando-se o programa EPINFO, baseados na população de cerca de mil pacientes, considerando prevalência de $5 \%$ e precisão de $4 \%$, chegou-se ao número de 88, aumentando-se para 105 ao se estimar as perdas em torno de $20 \%$.

Os pacientes foram selecionados por conveniência, à proporção que se apresentaram para as consultas, no período de janeiro a outubro de 2007, respeitando-se os critérios de exclusão do estudo, que foram: uso de beta-bloqueador, espironolactona, corticosteróides e anticoncepcionais, assim como gravidez.

Aos pacientes selecionados, foi aplicado um questionário, por meio do qual foram avaliadas as suas características demográficas e sintomatologia. Todos foram submetidos a exame físico com medidas da pressão arterial nos dois membros superiores e com os pacientes na posição sentada.

Todos realizaram exames laboratoriais, com dosagens de sódio e potássio séricos, creatinina, aldosterona sérica e atividade plasmática da renina, determinando-se a relação aldosterona/APR.

A coleta do sangue para dosagem da aldosterona e da APR foi realizada no período da manhã, com os pacientes em jejum e após 20 minutos de repouso na posição deitada, sem suspensão do tratamento anti-hipertensivo, e em sua dieta habitual. O sangue para dosagem da APR foi armazenado em tubos pré-refrigerados contendo ácido etileno-diaminotetraacético e o plasma foi separado imediatamente após a coleta em centrífuga refrigerada a 4 graus Celsius. A determinação da APR foi realizada por meio da geração de angiotensina I in vitro por radioimunoensaio, utilizando-se um kit comercialmente disponível, o Renin Maia ${ }^{\circledR}$ (Adaltis Itália, Itália). Os coeficientes de variação intra-ensaio e inter-ensaio variaram de 3,39 a $5,1 \%$ e 3,82 a $5,15 \%$, respectivamente, e os valores de normalidade para APR na posição deitada variaram de 0,51 a $2,64 \mathrm{ng} / \mathrm{ml} / \mathrm{h}$.

O sangue para dosagem da aldosterona foi armazenado em tubo plástico e o soro separado imediatamente após a coleta em centrífuga comum. A aldosterona sérica foi medida com a utilização de um radioimunoensaio de fase sólida baseado em anticorpos específicos da aldosterona imobilizados na parede de um tubo de polipropileno (Coat-A-Count Aldosterone, PITKAL ${ }^{\circledR}$ - 4, Los Angeles, USA). Os coeficientes de variação intra-ensaio e inter-ensaio tiveram variação de 2,3 a 5,4\% e 3,8 a 15,7\%, respectivamente, sendo que os valores de normalidade para aldosterona na posição deitada variaram de 1 a $16 \mathrm{ng} / \mathrm{dl}$.

As amostras de sangue para dosagem de APR e aldosterona foram armazenadas em freezer a uma temperatura de 20 graus Celsius negativos, pelo período de uma a duas semanas até serem enviadas para o laboratório central (Laboratório Hermes Pardini, Belo Horizonte, MG).

As amostras foram enviadas acondicionadas em gelo reciclável, em embalagem de isopor de cinco litros, de modo a manter a temperatura adequada durante todo o trajeto. $\mathrm{O}$ transporte foi realizado por via aérea, totalizando seis horas de viagem até o ponto final do destino.

A relação aldosterona/APR foi obtida através da divisão do valor da aldosterona sérica (ng/dl) pelo valor simultâneo da APR $(\mathrm{ng} / \mathrm{ml} / \mathrm{h})$, sendo definido um valor de $>25$ como teste de triagem positivo para o HAP.

Os pacientes que apresentaram a relação aldosterona/APR $>25$, realizaram teste de supressão com infusão de 2,0 litros de solução salina a 0,9\% por via endovenosa durante 4 horas (de 8:00h da manhã ao meio-dia), com dosagem da aldosterona sérica antes e depois da sobrecarga salina. A presença de aldosterona sérica $>05 \mathrm{ng} / \mathrm{dl}$ após a infusão da solução salina confirmou a autonomia da secreção da aldosterona, e consequentemente, o diagnóstico do HAP.

O passo seguinte consistiu em determinar a etiologia do HAP. Foi realizada tomografia computadorizada de adrenais com cortes de $03 \mathrm{~mm}$, antes e após a administração de contraste iodado, no paciente em que se confirmou a autonomia da secreção da aldosterona.

A presença de um macroadenoma adrenal solitário unilateral com supra-renal contralateral normal foi o critério adotado para diagnosticar adenoma produtor de aldosterona (APA).

Já a combinação dos critérios hormonais e bioquímicos descritos acima com tomografia computadorizada de adrenais mostrando hiperplasia micronodular bilateral, ou glândulas aparentemente normais, foi considerada critério diagnóstico de hiperplasia adrenocortical bilateral (HAB).

Os dados foram organizados e analisados através do programa EPINFO.

As variáveis quantitativas são apresentadas como médias e desvio-padrão, enquanto as variáveis qualitativas, como porcentagens. 
A pesquisa foi aprovada pelo Comitê de Ética em Pesquisa do Hospital Universitário e Termo de Consentimento Livre e Esclarecido foi obtido de todos os pacientes.

\section{Resultados}

No período de janeiro a outubro de 2007, foram incluídos 107 pacientes no protocolo de pesquisa do HAP, dos quais 105 realizaram os testes de triagem necessários para o diagnóstico.

Dos 105 pacientes, sete $(6,54 \%)$ apresentavam hipertensão arterial refratária. As características demográficas da amostra estudada são apresentadas na Tabela 1.

Todos os pacientes estavam recebendo terapia antihipertensiva e a freqüência dos medicamentos pode ser observada na Tabela 2.

Tabela 1 - Características demográficas dos 105 pacientes hipertensos incluídos no protocolo de pesquisa do hiperaldosteronismo primário, de janeiro a outubro de 2007, no Hospital Universitário da Universidade Federal do Maranhão

\begin{tabular}{lc}
\hline Sexo feminino (\%) & 75,2 \\
\hline Sexo masculino (\%) & 24,8 \\
\hline Idade (anos) & $55,1 \pm 11,2$ \\
\hline FC (bpm) & $74,4 \pm 9,5$ \\
\hline PAS (mmHg) & $138,1 \pm 20,1$ \\
\hline PAD (mmHg) & $83,4 \pm 11,7$ \\
\hline PA ótima, normal e limítrofe (\%) & 54,8 \\
\hline HAS Estágio I (\%) & 34,6 \\
\hline HAS Estágio II (\%) & 7,7 \\
\hline HAS Estágio III (\%) & 2,9 \\
\hline HAS Refratária (\%) & 6,54 \\
\hline Número de Anti-hipertensivos em uso & \\
\hline 1 (\%) & 26,17 \\
\hline $2(\%)$ & 58,88 \\
\hline 3 (\%) & 12,15 \\
\hline $4(\%)$ & 2,80 \\
\hline
\end{tabular}

FC - freqüência cardíaca; $P A S$ - pressão arterial sistólica; $P A D$ - pressão arterial diastólica; PA - pressão arterial; HAS - hipertensão arterial sistêmica.

Tabela 2 - Anti-hipertensivos em uso pelos 105 pacientes hipertensos incluídos no protocolo de pesquisa do hiperaldosteronismo primário, de janeiro a outubro de 2007, no Hospital Universitário da Universidade Federal do Maranhão

\begin{tabular}{lc}
\hline Anti-hipertensivos & $\%$ \\
\hline IECA & 78,5 \\
\hline Diuréticos tiazídicos & 69,0 \\
\hline Bloqueadores dos canais de cálcio & 23,3 \\
\hline Bloqueadores dos Receptores da Angiotensina II & 9,3 \\
\hline IECA - inibidores da enzima conversora de angiotensina.
\end{tabular}

Os parâmetros bioquímicos dos pacientes incluídos no protocolo da pesquisa podem ser observados na Tabela 3 . Dos 105 pacientes do estudo, nove (8,5\%) apresentaram potássio sérico menor que $3,5 \mathrm{mg} / \mathrm{dl}$ e outros nove $(8,5 \%)$ apresentaram relação aldosterona/APR > 25 (ng/dl)/(ng/ml/h), não sendo observada associação entre esses dois parâmetros, conforme mostra a Tabela 4.

$\mathrm{Na}$ Tabela 5 podem ser observados os valores de aldosterona sérica e APR dos nove pacientes com relação aldosterona/APR > 25 .

O teste de supressão com infusão salina foi realizado para a confirmação do HAP em oito dos nove pacientes com teste de triagem inicial positivo. Destes, apenas um (0,96\%), portador de hipertensão refratária, teve o diagnóstico de HAP confirmado. Nos outros sete pacientes, houve supressão da aldosterona, confirmando-se a presença de HAS essencial. Esses resultados podem ser observados na Tabela 6.

Foi realizada tomografia computadorizada de adrenais com cortes de $3 \mathrm{~mm}$ no paciente com diagnóstico sindrômico de HAP confirmado, sendo considerada normal, conforme mostra a figura 1 .

Tabela 3 - Parâmetros bioquímicos dos 105 pacientes hipertensos incluídos no protocolo de pesquisa do hiperaldosteronismo primário, de janeiro a outubro de 2007, no Hospital Universitário da Universidade Federal do Maranhão

\begin{tabular}{lc}
\hline Variável & Média \pm DP \\
\hline Creatinina sérica $(\mathrm{mg} / \mathrm{dl})$ & $0,8 \pm 0,2$ \\
\hline Potássio sérico $(\mathrm{mg} / \mathrm{dl})$ & $4,1 \pm 0,4$ \\
\hline Sódio sérico $(\mathrm{mg} / \mathrm{dl})$ & $141 \pm 2,9$ \\
\hline Aldosterona sérica $(\mathrm{ng} / \mathrm{dl})$ & $5,1 \pm 4,1$ \\
\hline Atividade plasmática da renina $(\mathrm{ng} / \mathrm{m} / \mathrm{h})$ & $1,8 \pm 2,0$ \\
\hline Relação aldosterona/APR $(\mathrm{ng} / \mathrm{d}) /(\mathrm{ng} / \mathrm{m} / \mathrm{h})$ & $8,1 \pm 11,9$ \\
\hline
\end{tabular}

$D P$ - desvio-padrão; $A P R$ - atividade plasmática da renina.

Tabela 4 - Associação entre potássio sérico e relação aldosteronal APR $>25$ nos pacientes hipertensos incluídos no protocolo de pesquisa do hiperaldosteronismo primário, de janeiro a outubro de 2007, no Hospital Universitário da Universidade Federal do Maranhão

\begin{tabular}{lcc}
\hline Paciente & $\mathrm{K}^{+}$Sérico (mg/dl) & $\begin{array}{c}\text { Relação Aldosterona/APR } \\
(\mathrm{ng} / \mathrm{dl}) /(\mathrm{ng} / \mathrm{ml} / \mathrm{h})\end{array}$ \\
\hline 1 & 3,9 & 52 \\
\hline 2 & 4,3 & 67 \\
\hline 3 & 4,2 & 34,5 \\
\hline 4 & 3,5 & 49 \\
\hline 5 & 4,2 & 27,5 \\
\hline 6 & 4,0 & 42,8 \\
\hline 7 & 3,8 & 25 \\
\hline 8 & 4,1 & 26,6 \\
\hline 9 & 4,1 & 39,5 \\
\hline
\end{tabular}

$K^{+}$- potássio; $A P R$ - atividade plasmática da renina. 


\section{Artigo Original}

Uma vez confirmada a autonomia da secreção da aldosterona, e consequentemente, o diagnóstico de HAP, foi iniciado o tratamento do paciente com espironolactona ( $25 \mathrm{mg} / \mathrm{dia})$.

\section{Discussão}

Neste estudo, foi utilizada a relação aldosterona/APR em 105 pacientes para pesquisa do HAP, e encontrou-se

Tabela 5 - Valores de aldosterona sérica e atividade plasmática da renina nos nove pacientes hipertensos do protocolo de pesquisa do hiperaldosteronismo primário que apresentaram relação aldosterona/APR $>25$ (janeiro a outubro de 2007, Hospital Universitário da Universidade Federal do Maranhão)

\begin{tabular}{lccc}
\hline Paciente & $\begin{array}{c}\text { Aldosterona } \\
\text { sérica (ng/dl) }\end{array}$ & APR (ng/ml/h) & $\begin{array}{c}\text { Relação } \\
\text { Aldosterona/APR } \\
\text { (ng/dl) / (ng/ml/h) }\end{array}$ \\
\hline 1 & 5,2 & 0,1 & 52 \\
\hline 2 & 6,7 & 0,1 & 67 \\
\hline 3 & 6,9 & 0,2 & 34,5 \\
\hline 4 & 4,9 & 0,1 & 49 \\
\hline 5 & 5,5 & 0,2 & 27,5 \\
\hline 6 & 21,4 & 0,5 & 42,8 \\
\hline 7 & 7,5 & 0,3 & 25 \\
\hline 8 & 8,0 & 0,3 & 26,6 \\
\hline 9 & 7,9 & 0,2 & 39,5 \\
\hline
\end{tabular}

APR - atividade plasmática da renina.

prevalência de $0,96 \%$ do total. No entanto, quando avaliados apenas os portadores de hipertensão arterial refratária, a prevalência do HAP foi de 14,3\%.

Apesar de vários estudos demonstrarem aumento significativo na prevalência do HAP com a utilização da relação aldosterona/APR, essa epidemia não encontra aceitação unânime no meio científico, sobretudo pela falta de padronização do teste de triagem ${ }^{6}$.

A relação aldosterona/APR sofre influência da postura, da dieta, e pode variar de alta a normal em estudos repetidos

Tabela 6 - Resultados do teste de supressão com solução salina endovenosa nos oito pacientes do protocolo de pesquisa do hiperaldosteronismo primário com relação aldosterona/APR $>25$ (janeiro a outubro de 2007, Hospital Universitário da Universidade Federal do Maranhão)

\begin{tabular}{lcc}
\hline Paciente & $\begin{array}{c}\text { Aldosterona Pré-Infusão } \\
\text { (ng/dl) }\end{array}$ & $\begin{array}{c}\text { Aldosterona Pós-Infusão } \\
\text { (ng/dl) }\end{array}$ \\
\hline 1 & 8,0 & 2,1 \\
\hline 2 & 6,8 & 0,5 \\
\hline 3 & 8,2 & 1,7 \\
\hline 4 & 13,1 & 6,6 \\
\hline 5 & 11,6 & 1,7 \\
\hline 6 & 7,0 & 3,0 \\
\hline 7 & 7,4 & 2,5 \\
\hline 8 & 9,9 & 2,8 \\
\hline
\end{tabular}

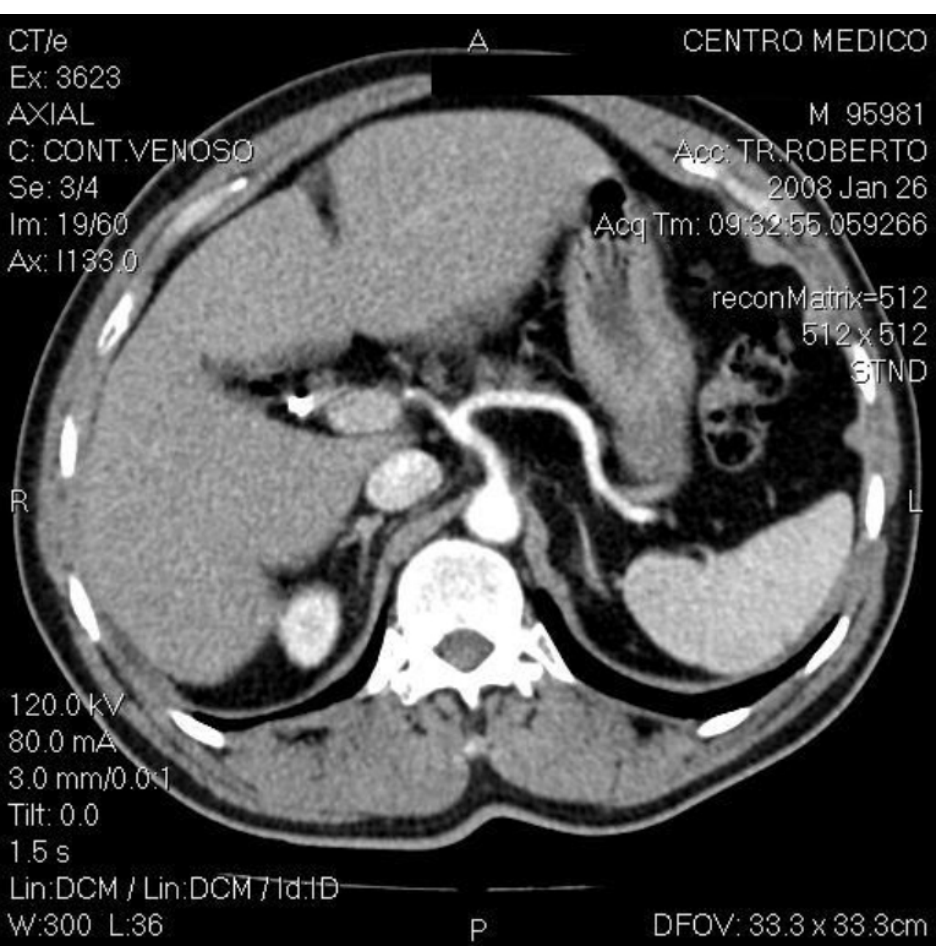

Fig. 1 - Tomografia computadorizada de supra-renais de paciente com diagnóstico de hiperaldosteronismo primário. 
em um mesmo indivíduo ${ }^{6}$.

No presente estudo, a coleta do sangue para dosagem de aldosterona e APR foi realizada pela manhã, com os pacientes em jejum, após repouso de 20 minutos na posição deitada, e em vigência do tratamento anti-hipertensivo usual. $O$ valor de corte da relação aldosterona/APR $>25$ foi adotado como positivo para a triagem do HAP.

Atualmente, a relação aldosterona/APR $>25$ tem sido utilizada como principal procedimento de rastreamento na investigação do $\mathrm{HAP}^{1}$. Giacchetti e cols. ${ }^{7}$, usando 25 como valor de corte para a relação aldosterona/APR obtida na posição deitada obtiveram sensibilidade de 98,3\% e especificidade de $67 \%$, valor preditivo positivo de $68 \%$ e negativo de $39 \%$.

Houve predomínio do sexo feminino na amostra estudada, refletindo a realidade dos consultórios, onde as mulheres estão mais presentes ${ }^{8}$.

A idade dos pacientes variou de 33 a 95 anos, com média de 55,1 anos, estando de acordo com o descrito por outros autores ${ }^{8-11}$.

Dos 105 pacientes, sete $(6,54 \%)$ eram portadores de hipertensão refratária, inclusive o paciente que teve o diagnóstico de HAP confirmado e encontrava-se em estágio II de HAS, apesar do uso de quatro classes diferentes de anti-hipertensivos (inibidores da enzima conversora da angiotensina (IECA), diurético tiazídico, bloqueador de canal de cálcio e simpaticolítico de ação central), sendo também diabético. Esse dado é concordante com a literatura médica; nela se observa que o HAP é mais comum entre os portadores de HAS refratária ${ }^{12}$.

Umpierrez e cols. ${ }^{11}$, estudando cem pacientes diabéticos e hipertensos refratários, encontraram prevalência de 14\% de HAP nessa população.

Todos os pacientes do estudo estavam recebendo terapia anti-hipertensiva, sendo que $78,5 \%$ estavam em uso de IECA e $69 \%$ recebiam diuréticos tiazídicos.

Sabe-se que os anti-hipertensivos são capazes de interferir na relação aldosterona/APR, sobretudo por suas influências sobre a APR.

De acordo com estudo conduzido por Mulatero e cols. ${ }^{13}$ em 230 pacientes, para avaliar os efeitos dos anti-hipertensivos sobre a relação aldosterona/APR, os alfa-bloqueadores e os IECA não interferem significativamente no diagnóstico do HAP. Já a amlodipina aumenta a taxa de falsos negativos e os beta-bloqueadores aumentam os falsos positivos.

A APR tende a ser mais baixa em pacientes usando betabloqueadores, o que aumenta o número de falsos positivos e a necessidade de testes confirmatórios, por isso foram excluídos os pacientes usuários desses medicamentos do estudo em questão.

Contudo, Nishizaka e cols. ${ }^{10}$, avaliando 265 pacientes hipertensos refratários com o objetivo de estabelecer a validade da relação aldosterona/APR em indivíduos brancos e negros, encontraram valores de corte da relação aldosterona/APR semelhantes para pacientes com e sem uso de beta-bloqueadores.

Mesmo com esse conhecimento, tem-se procurado não interferir com a medicação dos pacientes, reforçando a real filosofia de um teste de triagem ${ }^{14,15}$.

Hipocalemia não foi observada no paciente com HAP. Esse dado está em concordância com a maioria dos estudos em que a hipocalemia tem sido encontrada em número cada vez menor de pacientes com HAP (até 35\% dos casos) ${ }^{3,9}$.

Mosso e cols. ${ }^{8}$, estudando cem pacientes hipertensos com pressão arterial > $145 \times 95 \mathrm{mmHg}$, encontraram prevalência de $10 \%$ de HAP e nenhum paciente apresentava hipocalemia. Já Rossi e cols. ${ }^{16}$, em estudo com 1.125 pacientes hipertensos, encontraram prevalência de HAP de 4,8\%, com hipocalemia presente em 9,6\% dos pacientes.

A média da aldosterona da amostra estudada foi de 5,1 ng/ dl, e o paciente com HAP confirmado apresentou aldosterona sérica de 4,9 ng/dl na avaliação de triagem para a doença. Esses valores são considerados baixos quando comparados com os resultados de outros trabalhos da literatura. Umpierrez e cols. ${ }^{11}(n=100)$ encontraram valores médios de aldosterona de 9,1 $\pm 6 \mathrm{ng} / \mathrm{dl}$ nos hipertensos essenciais e 15,6 $\pm 8 \mathrm{ng} / \mathrm{dl}$ nos portadores de HAP. LOH e cols. ${ }^{9}(\mathrm{n}=350)$, também descreveram valores semelhantes, com média de 10,3 \pm $0,4 \mathrm{ng} / \mathrm{dl}$ em normotensos, 10,3 $\pm 0,4 \mathrm{ng} / \mathrm{dl}$ em hipertensos essenciais e 21,9 $\pm 1,1 \mathrm{ng} / \mathrm{dl}$ entre aqueles com $\mathrm{HAP}^{9}$. Resultados semelhantes também foram descritos por Mosso e cols. ${ }^{8}$ e Nishizaka e cols. ${ }^{10}$. Fogari e cols. ${ }^{17}$, avaliando três mil pacientes hipertensos não selecionados, obtiveram os seguintes valores médios de aldosterona: $8,1 \pm 3,8 \mathrm{ng} / \mathrm{dl}$ em hipertensos essenciais e 13,6 \pm 6,2 $\mathrm{ng} / \mathrm{dl}$ entre os portadores de HAP.

Williams e cols. ${ }^{18}$, em estudo com 347 pacientes hipertensos leves e moderados, apresentaram resultados similares aos do presente trabalho, com média de aldosterona sérica de 3,7 $\pm 0,2 \mathrm{ng} / \mathrm{dl}$ em normotensos e de 5,6 $\pm 0,2$ $\mathrm{ng} / \mathrm{dl}$ entre os hipertensos. Aldosterona média de 11,4 \pm $0,6 \mathrm{ng} / \mathrm{dl}$ foi encontrada entre aqueles com teste de triagem positivo para HAP.

Uma possível explicação para os baixos valores de aldosterona no trabalho mencionado é a ingestão excessiva de sódio pelos pacientes, uma vez que os resultados foram semelhantes aos de Williams e cols. ${ }^{18}(\mathrm{n}=347)$, em que os indivíduos estavam em dieta rica em sódio para a realização do teste de triagem para o HAP.

Já com relação à $A P R$, a média na presente pesquisa foi de $1,8 \mathrm{ng} / \mathrm{ml} / \mathrm{h}$, enquanto a do paciente com HAP confirmado foi de $0,1 \mathrm{ng} / \mathrm{ml} / \mathrm{h}$. A média geral é considerada alta em relação aos outros trabalhos da literatura. Loh e cols. ${ }^{9}(n=350)$ relataram resultados de 1,1 $\pm 0,10 \mathrm{ng} / \mathrm{ml} / \mathrm{h}$ entre os hipertensos essenciais e 0,29 $\pm 0,09 \mathrm{ng} / \mathrm{ml} / \mathrm{h}$ entre os portadores de HAP. Williams e cols. ${ }^{18}(\mathrm{n}=347)$ descreveram valores de $0,6 \pm 0,05 \mathrm{ng} / \mathrm{ml} / \mathrm{h}$ entre os hipertensos essenciais e 0,2 $\pm 0,02 \mathrm{ng} / \mathrm{ml} / \mathrm{h}$ entre aqueles com triagem positiva para HAP.

Nishizaka e cols. ${ }^{10}(\mathrm{n}=265)$, avaliando o efeito dos antihipertensivos sobre a APR, observaram que ela foi maior entre os usuários de IECA $(4,1 \pm 8,9 \times 3,0 \pm 6,2)$ e, sobretudo, entre aqueles em uso de diuréticos tiazídicos $(4,0 \pm 8,5 \times 1,6 \pm$ $2,7)$, onde a diferença foi estatisticamente significativa.

O fato da grande maioria dos pacientes do estudo estar em uso de IECA em associação com diurético tiazídico pode justificar a elevação da APR. 
A média da relação aldosterona/APR no presente trabalho foi de $8,1(\mathrm{ng} / \mathrm{dl}) /(\mathrm{ng} / \mathrm{ml} / \mathrm{h})$, e a relação do paciente com HAP confirmado foi de 49 (ng/dl) / (ng/ml/h). Loh e cols. $^{9}(n=350)$ encontraram média de 19,4 $\pm 1,2$ $(\mathrm{ng} / \mathrm{dl}) /(\mathrm{ng} / \mathrm{ml} / \mathrm{h})$ entre os hipertensos essenciais e de 484 $\pm 128,5(\mathrm{ng} / \mathrm{dl}) /(\mathrm{ng} / \mathrm{ml} / \mathrm{h})$ entre os portadores de HAP. Já Williams e cols. ${ }^{18}(\mathrm{n}=347)$ relataram média de 14,8 $\pm 0,8$ $(\mathrm{ng} / \mathrm{dl}) /(\mathrm{ng} / \mathrm{ml} / \mathrm{h})$ entre os hipertensos essenciais e de 64,8 $\pm 6(\mathrm{ng} / \mathrm{dl}) /(\mathrm{ng} / \mathrm{ml} / \mathrm{h})$ entre aqueles com teste de triagem positivo para HAP.

Na presente pesquisa, nove pacientes (8,5\%), apresentaram relação aldosterona/APR > $25(\mathrm{ng} / \mathrm{dl}) /(\mathrm{ng} / \mathrm{ml} / \mathrm{h})$, dos quais oito foram submetidos ao teste de supressão com sobrecarga salina E.V. (endovenosa) para confirmação do HAP, o que se demonstrou em apenas um paciente hipertenso refratário (0,96\% do total e 14,3\% dos hipertensos refratários).

Os sete pacientes com relação aldosterona/APR > 25 (ng/dl) / (ng/ml/h) que tiveram a aldosterona sérica suprimida após a sobrecarga salina foram considerados portadores de HAS essencial com renina baixa.

O uso da relação aldosterona/APR é passível de muitas críticas. Por exemplo, valores baixos e até indetectáveis de renina são comuns em pacientes idosos, e hipertensos de longa data, aumentando a relação aldosterona/APR, mesmo em presença de aldosterona sérica normal.

Alguns serviços como os de Torino (Itália), Rochester (Minnesota) e Singapura, adotam a relação aldosterona/APR associada a um valor mínimo de aldosterona sérica para triagem do HAP com o objetivo de diminuir o número de falsos positivos ${ }^{3}$. Mulatero e cols. ${ }^{19}$ consideram a relação aldosterona/APR elevada em associação com aldosterona sérica maior que $15 \mathrm{ng} / \mathrm{dl}$ como método de triagem ideal para HAP. Já Passos e cols. ${ }^{14}$ advertem que a relação aldosterona/ APR deverá ser interpretada com cautela quando os valores de aldosterona estiverem abaixo de $12 \mathrm{ng} / \mathrm{dl}$. Por outro lado, em Brisbane (Austrália), não se estabelece valor de corte para aldosterona sérica, assim como em Santiago (Chile) $)^{3,20}$. Os resultados são semelhantes quanto à prevalência do HAP em todos esses centros ${ }^{3}$.

Um estudo com 118 pacientes hipertensos e normocalêmicos realizado na Mayo Clinic detectou que 30\% deles apresentavam elevação da relação aldosterona/APR, mas somente 13\% tinham excreção de aldosterona também elevada ${ }^{21}$.

No presente estudo, foi adotado o valor de corte $>25$ para triagem positiva para o HAP, independente dos valores de aldosterona, priorizando-se a maior sensibilidade do teste.

De acordo com dados do Princess Alexandra Hospital, em Brisbane, apenas 19\% dos pacientes que apresentaram lateralização detectada pelo cateterismo seletivo de veias supra-renais tinham aldosterona sérica $>15 \mathrm{ng} / \mathrm{dl}$ e muitos diagnósticos de HAP seriam perdidos se o valor mínimo de aldosterona sérica fosse adotado como parte do teste de triagem para a patologia ${ }^{20}$.

Apesar do maior número de falsos positivos ao eliminarmos o valor mínimo de aldosterona sérica na triagem do HAP, eles serão facilmente diferenciados dos verdadeiros positivos por meio dos testes de supressão ${ }^{11}$.
Sabe-se que a relação aldosterona/APR isolada não é suficiente para o diagnóstico do HAP. Menos de $50 \%$ daqueles com relação aldosterona/APR elevada falham em suprimir os níveis de aldosterona plasmática ou urinária após sobrecarga salina oral ou endovenosa ${ }^{22}$.

Séries de diversos autores revelam percentual elevado de pacientes com relação aldosterona/APR aumentada, que, após a realização do teste de supressão, é reduzido para valores comparáveis às prevalências anteriores do HAP6. Portanto, os testes de supressão são obrigatórios para o diagnóstico do $\mathrm{HAP}^{10}$.

No presente estudo, foi realizado teste de supressão com a infusão de 2 litros de soro fisiológico a 0,9\% E.V. em quatro horas. Considerou-se a presença de autonomia na secreção de aldosterona, uma aldosterona sérica > $5 \mathrm{ng} / \mathrm{dl}$ após a administração da solução salina, valor adotado em outros trabalhos publicados na literatura ${ }^{5,23}$

Apenas um paciente, $(0,96 \%$ do total e $14,3 \%$ dos hipertensos refratários) teve confirmada a secreção autônoma de aldosterona e, consequentemente, o HAP $(13,1 \mathrm{ng} /$ dl x 6,6 ng/dl, aldosterona pré-infusão e pós-infusão, respectivamente).

Para diferenciação etiológica do HAP, foi realizada tomografia computadorizada de adrenais com cortes de $3 \mathrm{~mm}$. O exame foi considerado normal. Esse resultado é concordante com o estudo da Mayo Clinic, em que dentre os pacientes hipertensos e normocalêmicos com teste de triagem positivo para HAP, nenhum apresentou tumorações em supra-renais na tomografia computadorizada ${ }^{21}$.

Isso pode ser explicado pela alta incidência de hiperplasia adrenal detectável apenas pelo exame histológico da glândula. Assim, a ausência de substrato radiológico não invalida o diagnóstico de HAP se as evidências bioquímicas são conclusivas ${ }^{8}$.

O exame considerado padrão-ouro para diferenciar os tumores das hiperplasias de supra-renais é o cateterismo seletivo das veias supra-renais com coleta de sangue para dosagem de aldosterona e cortisol. Trata-se de método diagnóstico invasivo, caro, não disponível em muitos centros e tecnicamente difícil de ser realizado. Por isso, não foi realizado no estudo em questão.

Dentre as limitações da pesquisa, pode-se citar o fato da amostra ter sido selecionada por conveniência e em um hospital universitário. Apesar da inclusão de forma consecutiva, a seleção dos pacientes em instituições como hospitais não envolve indivíduos de outra clientela e os resultados refletem a experiência do próprio serviço, não podendo ser generalizados para a comunidade e para a prática clínica em consultórios. Não se pode esquecer, ainda, o fato de a triagem ter sido realizada na vigência do tratamento anti-hipertensivo, com todas as possíveis influências dos medicamentos sobre a APR, sobretudo dos IECA em associação com o diurético tiazídico. Essa associação pode ter sido responsável pela elevação da APR, gerando resultados falsos negativos.

Quanto à relevância clínica do estudo, destaca-se a sua originalidade, uma vez que não existem publicações locais nem regionais sobre o assunto. 


\section{Conclusão}

A prevalência do HAP na Liga de HAS do Hospital Universitário do estudo, considerando-se toda a amostra, foi de $0,96 \%$. No entanto, quando avaliados apenas os portadores de hipertensão arterial refratária, a prevalência atinge cifras de $14,3 \%$. Finalmente, ao se avaliar os prós e os contras da utilização da relação aldosterona/APR como método de triagem para o HAP, sobretudo a falta de padronização para a sua realização, a falta de um valor de corte estabelecido, a influência que sofre da APR (e de todas as variáveis que a alteram: sexo, idade, HAS, medicamentos), além da sua insuficiência como teste diagnóstico, levando à necessidade da realização de outros exames mais dispendiosos e invasivos, conclui-se que ela não deve ser utilizada de rotina para avaliação indiscriminada de todos os hipertensos. Deve-se proceder

\section{Referências}

1. Kater CE. Hiperaldosteronismo primário: novas tendências. Rev Bras Hipertens., 2002; 9: 165-73.

2. Young WF Jr. Primary aldosteronism: management issues. Ann N Y Acad Sci. 2002; 970: 61-76.

3. Mulatero P, Stowasser M, Loh KC, Fardella CE, Gordon RD, Mosso L, et al. Increased diagnosis of primary aldosteronism, including surgically correctable forms, in centers from five continents. J Clin Endocrinol Metab. 2004; 89: 1045-50.

4. Francishetti EA, Abreu VG. Investigações diagnósticas em hipertensão arterial - hiperaldosteronismo. In: Sociedade Brasileira de Cardiologia. I.I Hipertensão arterial: programa de educação continuada. Rio de Janeiro: Diagraphic; 2002. p. 6-7.

5. Gomez-Hernández K, Chen-Ku CH. Hiperaldosteronismo primario, una nueva perspectiva. Acta Méd Costarric. 2007; 49: 12-20.

6. Kaplan NM. Is there an unrecognized epidemic of primary aldosteronism? (Con). Hypertension. 2007; 50: 454-8.

7. Giacchetti G, Ronconi V, Lucarelli G, Boscaro M, Mantero F. Analysis of screening and confirmatory tests in the diagnosis of primary aldosteronism: need for a standardized protocol. J Hypertens. 2006; 24: 737-45.

8. Mosso LG, Fardella C, Montero J, Rojas P, Sánchez O, Rojas V, et al. Alta prevalência de hiperaldosteronismo primario no diagnosticado en hipertensos catalogados como esenciales. Rev Méd Chil. 1999; 127 : 800-6.

9. Loh KC, Koay ES, Khaw MC, Emmanuel SC, Young WF Jr. Prevalence of primary aldosteronism among asian hypertensive patients in Singapore. $J$ Clin Endocrinol Metab. 2000; 85: 2854-9.

10. Nishizaka MK, Ubunama MP, Zaman MA, Cofield S, Calhoun DA. Validity of plasma aldosterone-to-renin activity ratio in african american and white subjects with resistant hypertension. Am J Hypertens. 2005; 18: 805-12.

11. Umpierrez GE, Cantey P, Smiley D, Palacio A, Temponi D, Luster K, et al. Primary aldosteronism in diabetic subjects with resistant hypertension. Diabetes Care. 2007; 30: 1699-703. à pesquisa do HAP em pacientes hipertensos com hipocalemia, refratários ao tratamento usual e naqueles nos quais se encontram incidentalomas.

\section{Potencial Conflito de Interesses}

Declaro não haver conflito de interesses pertinentes.

\section{Fontes de Financiamento}

O presente estudo não teve fontes de financiamento externas.

\section{Vinculação Acadêmica}

Este artigo é parte de tese de Mestrado de Maria Jacqueline Silva Ribeiro pela Universidade Federal do Maranhão.
12. Calhoun DA. Is there an unrecognized epidemic of primary aldosteronism? (Pro). Hypertension. 2007; 50: 447-53.

13. Mulatero, P.; Rabbia, F.; Milan, A.; Paglieri, C.; Morello, F.; Chiandussi, L. et al. Drug effects on aldosterone/plasma renin activity ratio in primary aldosteronism. Hypertension. 2002; 40: 897-908.

14. Passos VQ, Martins LAL, Pereira MAA, Kater CE. Hiperaldosteronismo primário revisitado. Arq Bras Endocrinol Metab. 2001; 45: 285-301.

15. Kater CE. Hiperaldosteronismo primário. Arq Bras Endocrinol Metab. 2002 46: 106-15.

16. Rossi GP, Bernini G, Caliumi C, Desideri G, Fabris B, Ferri C, et al. A prospective study of the prevalence of primary aldosteronism in 1125 hypertensive patients. J Am Coll Cardiol. 2006; 48: 2293-300.

17. Fogari R, Preti P, Zoppi A, Rinaldi A, Fogari E, Mugellini A. Prevalence of primary aldosteronism among unselected hypertensive patients: a prospective study base don the use of an aldosterone/renin ratio above 25 as a screening test. Hypertens Rev. 2007; 30: 111-7.

18. Williams JS, Williams GH, Raji A, Jeunemaitre X, Brown NJ, Hopkins PN, et al. Prevalence of primary hyperaldosteronism in mild to moderate hypertension without hypokalemia. J Hum Hypertens. 2006; 20: 129-36.

19. Mulatero P, Dluhy RG, Giacchetti G, Boscaro M, Veglio F, Stewart PM. Diagnosis of primary aldosteronism: from screening to subtype differentiation. Trends Endocrinol Metab. 2005; 16: 114-9.

20. Stowasser M, Gordon RD. Primary aldosteronism - careful investigation is essential and rewarding. Mol Cell Endocrinol. 2004; 217: 33-9.

21. Krakoff LR. Screening for primary aldosteronism: progress and frustration. J Hypertens. 2006; 24: 635-7.

22. Kaplan NM. The current epidemic of primary aldosteronism: causes and consequences. J Hypertens. 2004; 22: 863-9.

23. Tiu SC, Choi CH, Shek CC, Ng YW, Chan FKW, Ng CM, et al. The use of aldosterone - renin ratio as a diagnostic test for primary hyperaldosteronism and its test characteristics under different conditions of blood sampling. J Clin Endocrinol Metab. 2005; 90: 72-8. 